\title{
Non-Line-of-Sight Mobile Station Positioning Algorithm using TOA, AOA, and Doppler-Shift
}

\author{
Rohan Ramlall, Jie Chen, Arnold Lee Swindlehurst \\ Department of Electrical Engineering and Computer Science \\ University of California, Irvine \\ Irvine, CA, 92697, USA \\ \{rramlall, jie.chen, swindle\}@uci.edu
}

\begin{abstract}
Location estimation in wireless communications systems experiencing purely non-line-of-sight propagation paths is an open problem. In this paper, a novel least squares algorithm is presented to estimate the location of a mobile station (MS) experiencing purely non-line-of-sight communication with at least one base station (BS) by using the time of arrival (TOA), angle of arrival (AOA), and Doppler-shift (DS) measurements collected at the BS. Simulation results are provided to demonstrate the estimation accuracy and improvement over existing methods.
\end{abstract}

Keywords-Enhanced 911, localization, positioning, non-lineof-sight propagation

\section{INTRODUCTION}

Location estimation in wireless communications systems experiencing purely non-line-of-sight (NLOS) propagation paths is an open problem. This is due to the fact that multipath is one of the major sources of error for location estimation because of the bias it introduces in the time of arrival (TOA) estimate. Many techniques in the literature have assumed the presence of both line-of-sight (LOS) and NLOS propagation paths between the base station (BS) and mobile station (MS), and have tried to identify and discard the NLOS paths; thus using only the LOS paths for location estimation. However, these techniques do not work in purely NLOS environments, which are typically experienced by wireless communication systems [1], where no LOS paths exist between the BS and MS.

Recently, techniques (e.g., [2]-[3]) have been presented to estimate the MS location in purely NLOS environments. These techniques utilize angle of arrival (AOA) and/or Doppler-shift (DS) measurements in addition to TOA measurements, all of which are collected at the BS. However, these techniques only perform well when extremely accurate measurements are available.

In this paper, a novel least squares (LS) algorithm is presented to estimate the location of a MS experiencing purely NLOS communication with at least one BS by using the TOA, AOA, and DS measurements collected at the BS. The proposed algorithm consists of two steps: in the first step, the location of the scatterers and speed of the MS are estimated using LS; in the second step, the location of the MS is estimated using nonlinear LS. The velocity of the MS can also be estimated using LS in an additional step. The key novelty of the proposed algorithm is the system of linear equations used to solve for the location of the scatterers and speed of the MS in the first step. The layout of the paper is as follows. Section II presents the system model and Section III presents the proposed algorithm. Section IV presents simulation results that demonstrate the performance of the proposed algorithm in picocellular, microcellular, and macrocelluar environments. The paper is concluded in Section V.

\section{SYSTEM MODEL}

One BS observes $N$ uplink communication signals from one MS. The MS is assumed to move at a constant velocity $v$ for a period of time $N \Delta t$ (which is small, where $\Delta t$ is the time between signal transmissions) while the $N$ uplink signals are transmitted. Each of the $N$ uplink signals arrives at the BS via $S$ distinct paths due to the presence of $S$ scatterers. It is assumed that all NS signals undergo a single-bounce reflection with one scatterer before arriving at the BS. Here, the term reflection can refer to both specular and diffuse reflections. Any signals that undergo multiple bounces are assumed to be attenuated enough that their contribution at the BS is negligible. Hence, there is no line-of-sight communication path between the BS and MS. This agrees with many wireless channel models proposed in the literature [1]. It is also assumed that the clocks on the BS and MS are synchronized in time and frequency. The position of the BS is assumed to be fixed and known, and the positions of the scatterers are assumed to be fixed and unknown. Both the MS position and velocity are assumed to be unknown. A two-dimensional picture of a possible system is shown in Fig. 1 with $S=3$. Note that the algorithm can readily be extended to cases with more than one BS.

The BS measures the time of arrival $\tau$, angle of arrival $\theta$, and Doppler-shift $\varsigma$ of all NS signals. For notational simplicity, let us first look at the measurements obtained from one BS and one scatterer. From geometry, the distance between the scatterer and BS is given by 


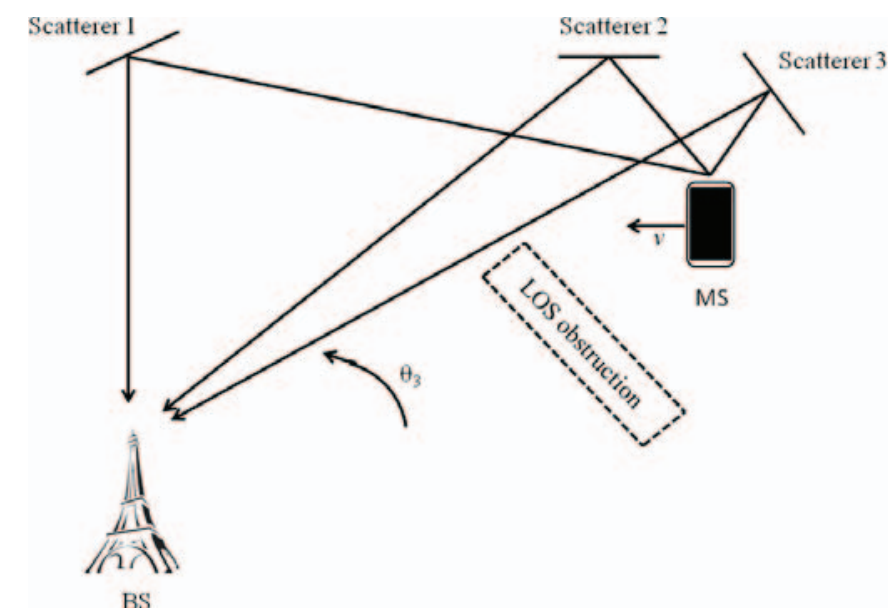

Fig. 1. Localization scenario with one BS and three scatterers

$$
d_{s c, b s}=\frac{x_{s c}-x_{b s}}{\cos \theta}=\frac{y_{s c}-y_{b s}}{\sin \theta}
$$

where $\left[\begin{array}{ll}x_{s c} & y_{s c}\end{array}\right]^{\mathrm{T}}$ is the position of the scatterer, $\left[x_{b s} y_{b s}\right]^{\mathrm{T}}$ is the position of the $\mathrm{BS}$, and $\theta$ is the mean of the $N$ AOA measurements of the signals arriving at the BS after reflecting off the scatterer. Cross multiplying the terms in (1) produces

$$
x_{s c} \sin \theta-y_{s c} \cos \theta=x_{b s} \sin \theta-y_{b s} \cos \theta
$$

The TOA measurement at time $n \Delta t$ of the signal arriving at the $\mathrm{BS}$ after reflecting off the scatterer is given by

$$
c \tau(n \Delta t)=\sqrt{\left(x_{s c}-x_{m s}(n \Delta t)\right)^{2}+\left(y_{s c}-y_{m s}(n \Delta t)\right)^{2}}+\sqrt{\left(x_{s c}-x_{b s}\right)^{2}+\left(y_{s c}-y_{b s}\right)^{2}}
$$

for $n=0, \ldots, N-1$, where $c$ is the propagation speed of the uplink signal $\left(3 \times 10^{8} \mathrm{~m} / \mathrm{s}\right)$ and $\left[x_{m s}(n \Delta t) y_{m s}(n \Delta t)\right]^{\mathrm{T}}$ is the position of the MS at time $n \Delta t$.

The DS measurement at time $n \Delta t$ of the signal arriving at the BS after reflecting off the scatterer is given by

$$
\begin{aligned}
\varsigma(n \Delta t) & =f_{R}(n \Delta t)-f_{T} \\
& =\frac{f_{T}}{c} \frac{v_{x}\left(x_{s c}-x_{m s}(n \Delta t)\right)+v_{y}\left(y_{s c}-y_{m s}(n \Delta t)\right)}{\sqrt{\left(x_{s c}-x_{m s}(n \Delta t)\right)^{2}+\left(y_{s c}-y_{m s}(n \Delta t)\right)^{2}}}
\end{aligned}
$$

for $n=0, \ldots, N-1$, where $f_{R}$ is the frequency of the received signal, $f_{T}$ is the frequency of the transmitted signal, and $\left[\begin{array}{ll}v_{x} & v_{y}\end{array}\right]^{\mathrm{T}}$ is the velocity of the MS. Since the BS and scatterer are both stationary, the DS observed at the BS is the same as the DS observed at the scatterer.

\section{LEAST SQUARES ALGORITHM}

Utilizing (3), (4) can be written as

$\varsigma(n \Delta t)=\frac{f_{T}}{c} \frac{v_{x}\left(x_{s c}-\left(x_{m s}(0)+v_{x} n \Delta t\right)\right)+v_{y}\left(y_{s c}-\left(y_{m s}(0)+v_{y} n \Delta t\right)\right)}{c \tau(n \Delta t)-d_{s c, b s}}$
Substituting (1) into (5), and after some simplification, (5) can be written as

$$
\begin{gathered}
-2 \frac{f_{T}}{c} v_{x} x_{m s}(0)-2 \frac{f_{T}}{c} v_{y} y_{m s}(0)+\left(\varsigma(n \Delta t) \sec \theta+2 \frac{f_{T}}{c} v_{x}\right) x_{s c} \\
+\left(\varsigma(n \Delta t) \csc \theta+2 \frac{f_{T}}{c} v_{y}\right) y_{s c}= \\
\left(2 c \tau(n \Delta t)+x_{b s} \sec \theta+y_{b s} \csc \theta\right) \varsigma(n \Delta t)+2 \frac{f_{T}}{c} n \Delta t\left(v_{x}^{2}+v_{y}^{2}\right)
\end{gathered}
$$

Subtracting (6) at $n=0$ from (6) at $n=1, \ldots, N-1$ results in

$$
\begin{gathered}
\sec \theta(\varsigma(n \Delta t)-\varsigma(0)) x_{s c}+\csc \theta(\varsigma(n \Delta t)-\varsigma(0)) y_{s c}-2 \frac{f_{T}}{c} n \Delta t\left(v_{x}^{2}+v_{y}^{2}\right)= \\
\left(2 c \tau(n \Delta t)+x_{b s} \sec \theta+y_{b s} \csc \theta\right) \varsigma(n \Delta t)- \\
\left(2 c \tau(0)+x_{b s} \sec \theta+y_{b s} \csc \theta\right) \varsigma(0)
\end{gathered}
$$

for $n=1, \ldots, N-1$.

Equations (2) and (7) can be combined for $S$ scatterers to form a linear model as follows. For scatterer $i$, define $\mathbf{A}_{\mathrm{i}}$ and $\mathbf{b}_{\mathrm{i}}$ as (see (9) on the next page)

$\mathbf{A}_{i}=\left[\begin{array}{cc}\sin \theta_{i} & -\cos \theta_{i} \\ \sec \theta_{i}\left(\varsigma_{i}(\Delta t)-\varsigma_{i}(0)\right) & \csc \theta_{i}\left(\varsigma_{i}(\Delta t)-\varsigma_{i}(0)\right) \\ \vdots & \vdots \\ \sec \theta_{i}\left(\varsigma_{i}((N-1) \Delta t)-\varsigma_{i}(0)\right) & \csc \theta_{i}\left(\varsigma_{i}((N-1) \Delta t)-\varsigma_{i}(0)\right)\end{array}\right]$

Also define

$$
\mathbf{A}_{v}=\left[\begin{array}{c}
0 \\
-2 \frac{f_{T}}{c} \Delta t \\
\vdots \\
-2 \frac{f_{T}}{c}(N-1) \Delta t
\end{array}\right]
$$

so that the linear model is given by

$$
\mathbf{A s}=\mathbf{b}
$$

where

$$
\mathbf{A}=\left[\begin{array}{ccccc}
\mathbf{A}_{1} & & & & \mathbf{A}_{v} \\
& \mathbf{A}_{2} & & & \mathbf{A}_{v} \\
& & \ddots & & \vdots \\
& & & \mathbf{A}_{S} & \mathbf{A}_{v}
\end{array}\right]
$$




$$
\mathbf{b}_{i}=\left[\begin{array}{c}
x_{b s} \sin \theta_{i}-y_{b s} \cos \theta_{i} \\
\left(2 c \tau_{i}(\Delta t)+x_{b s} \sec \theta_{i}+y_{b s} \csc \theta_{i}\right) \varsigma_{i}(\Delta t)-\left(2 c \tau_{i}(0)+x_{b s} \sec \theta_{i}+y_{b s} \csc \theta_{i}\right) \varsigma_{i}(0) \\
\vdots \\
\left(2 c \tau_{i}((N-1) \Delta t)+x_{b s} \sec \theta_{i}+y_{b s} \csc \theta_{i}\right) s_{i}((N-1) \Delta t)-\left(2 c \tau_{i}(0)+x_{b s} \sec \theta_{i}+y_{b s} \csc \theta_{i}\right) \varsigma_{i}(0)
\end{array}\right]
$$

$$
\begin{gathered}
\mathbf{s}=\left[\begin{array}{c}
x_{s c, 1} \\
y_{s c, 1} \\
x_{s c, 2} \\
y_{s c, 2} \\
\vdots \\
x_{s c, S} \\
y_{s c, S} \\
v_{x}^{2}+v_{y}^{2}
\end{array}\right] \\
\mathbf{b}=\left[\begin{array}{c}
\mathbf{b}_{1} \\
\mathbf{b}_{2} \\
\vdots \\
\mathbf{b}_{S}
\end{array}\right]
\end{gathered}
$$

Note that all of the entries in $\mathbf{A}$ besides the entries shown are 0 . From (11), the least squares estimate of the position of the $S$ scatterers and squared magnitude of the MS velocity is given by

$$
\hat{\mathbf{s}}=\left(\mathbf{A}^{\mathrm{T}} \mathbf{A}\right)^{-1} \mathbf{A}^{\mathrm{T}} \mathbf{b}
$$

Now that the position of the scatterers has been estimated, the position of the MS can be estimated at any time $n \Delta t$ for $n=$ $0, \ldots, N-1$ as follows. The distance between the MS and scatterer $i$ at time $n \Delta t$ is given by

$$
d_{i}(n \Delta t)=c \tau_{i}(n \Delta t)-\sqrt{\left(x_{b s}-\hat{x}_{s c, i}\right)^{2}+\left(y_{b s}-\hat{y}_{s c, i}\right)^{2}}
$$

Since the signals experience line-of-sight propagation from the MS to the scatterers and estimates of both the scatterer positions and distance between the MS and scatterers are available, the problem is now that of a conventional TOA positioning problem. For the results presented in this paper, the authors utilized the conventional iterative technique based on Taylor Series expansion [4] around an initial MS position estimate equal to the position of the BS.

In the subsequent simulation results, the iterative technique is considered to have diverged if more than 20 iterations are required for the 2-norm of the difference in consecutive MS position estimates to be less than $1 \mathrm{~cm}$.

Finally, if desired, the scatterer position estimates from (15) and the MS position estimates from above can be substituted into (4) to solve for the MS velocity using LS.

\section{Simulation Results}

The performance of the proposed algorithm is demonstrated through Monte Carlo simulations with 1000 realizations. The proposed algorithm is compared to the algorithm from [2], since it is the only other method that utilizes TOA, AOA, and DS measurements in an overdetermined system of equations. The initial position of the MS is $\left[x_{m s}(0) y_{m s}(0)\right]^{\mathrm{T}}=[5000 \mathrm{~m} 5000 \mathrm{~m}]^{\mathrm{T}}$; and the positions of the base station and scatterers are chosen according to an independent and identically distributed (i.i.d.) Gaussian random variable with mean $5000 \mathrm{~m}$ and standard deviation depending on if the cellular environment is a picocell, microcell, or macrocell (see Table I). The velocity of the MS is $\left[\begin{array}{ll}v_{x} & v_{y}\end{array}\right]^{\mathrm{T}}=[0.6 \mathrm{~m} / \mathrm{s}-0.8 \mathrm{~m} / \mathrm{s}]^{\mathrm{T}}$ in the picocellular environment and $\left[\begin{array}{ll}v_{x} & v_{y}\end{array}\right]^{\mathrm{T}}=[10 \mathrm{~m} / \mathrm{s} \quad 18 \mathrm{~m} / \mathrm{s}]^{\mathrm{T}}$ in the microcellular and macrocellular environments. $\Delta t=50 \mathrm{~ms}$ and $f_{T}=2.1 \mathrm{GHz}$.

TABLE I. CELlular ENVIRONMENT

\begin{tabular}{|c|c|c|}
\hline & $\sigma_{B S}$ & $\sigma_{S C}$ \\
\hline Picocell & $100 \mathrm{~m}$ & $100 \mathrm{~m}$ \\
\hline Microcell & $1 \mathrm{~km}$ & $700 \mathrm{~m}$ \\
\hline Macrocell & $10 \mathrm{~km}$ & $1 \mathrm{~km}$ \\
\hline
\end{tabular}

Tables II and III show the standard deviation of the measurement errors that are required to achieve the accuracy mandated by the Enhanced 911 positioning requirement of less than $50 \mathrm{~m}$ accuracy in $67 \%$ of positioning attempts [5] for $1 \mathrm{BS}$ and $S=3$ scatterers. Note the following:

- The aforementioned Enhanced 911 requirement applies to user equipment (UE)-based positioning methods, whereas the proposed algorithm is a network-based method whose Enhanced 911 requirement is less than $100 \mathrm{~m}$ accuracy in $67 \%$ of positioning attempts. However, the authors feel that the more stringent requirements for UE-based methods will provide better insight into the feasibility of the proposed algorithm for future localization systems where higher degrees of accuracy will be desired.

- In the picocellular environments, the results shown are for an accuracy of less than $10 \mathrm{~m}$ in $67 \%$ of positioning attempts, since $50 \mathrm{~m}$ level accuracy in picocells does not convey much information about the location of the MS. 
TABLE II. MEASUREMENT ERROR, 3 SCATtERERS, $N=100$

\begin{tabular}{|c|c|c|c|c|c|c|c|c|c|}
\hline & \multicolumn{3}{|c|}{ Picocell } & \multicolumn{3}{|c|}{ Microcell } & \multicolumn{3}{|c|}{ Macrocell } \\
\hline & $\sigma_{\tau}$ & $\sigma_{\theta}$ & $\sigma_{\varsigma}$ & $\sigma_{\tau}$ & $\sigma_{\theta}$ & $\sigma_{\varsigma}$ & $\sigma_{\tau}$ & $\sigma_{\theta}$ & $\sigma_{\varsigma}$ \\
\hline $\begin{array}{l}\text { Proposed } \\
\text { Algorithm }\end{array}$ & $1.3 \mathrm{ps}$ & $41^{\circ}$ & $10 \mu \mathrm{Hz}$ & $60 \mathrm{ps}$ & $22^{\circ}$ & $1 \mathrm{mHz}$ & $50 \mathrm{ps}$ & $2^{\circ}$ & $1 \mathrm{mHz}$ \\
\hline $\begin{array}{l}\text { Algorithm } \\
\text { from [2] }\end{array}$ & $0.6 \mathrm{ps}$ & $24^{\circ}$ & $500 \mathrm{nHz}$ & $20 \mathrm{ps}$ & $11^{\circ}$ & $80 \mu \mathrm{Hz}$ & $20 \mathrm{ps}$ & $1^{\circ}$ & $40 \mu \mathrm{Hz}$ \\
\hline
\end{tabular}

TABLE III. MEASUREMENT ERROR, 3 SCATtERERS, $N=200$

\begin{tabular}{|c|c|c|c|c|c|c|c|c|c|}
\hline & \multicolumn{3}{|c|}{ Picocell } & \multicolumn{3}{|c|}{ Microcell } & \multicolumn{3}{|c|}{ Macrocell } \\
\hline & $\sigma_{\tau}$ & $\sigma_{\theta}$ & $\sigma_{\varsigma}$ & $\sigma_{\tau}$ & $\sigma_{\theta}$ & $\sigma_{\varsigma}$ & $\sigma_{\tau}$ & $\sigma_{\theta}$ & $\sigma_{\varsigma}$ \\
\hline $\begin{array}{c}\text { Proposed } \\
\text { Algorithm }\end{array}$ & $5 p s$ & $57^{\circ}$ & $50 \mu \mathrm{Hz}$ & $200 \mathrm{ps}$ & $28^{\circ}$ & $7 \mathrm{mHz}$ & $200 \mathrm{ps}$ & $2^{\circ}$ & $3 \mathrm{mHz}$ \\
\hline $\begin{array}{l}\text { Algorithm } \\
\text { from [2] }\end{array}$ & $2 \mathrm{ps}$ & $34^{\circ}$ & $1 \mu \mathrm{Hz}$ & $90 \mathrm{ps}$ & $16^{\circ}$ & $100 \mu \mathrm{Hz}$ & $90 \mathrm{ps}$ & $1^{\circ}$ & $80 \mu \mathrm{Hz}$ \\
\hline
\end{tabular}

TABLE IV. MEASUREMENT ERROR, 2 SCATTERERS

\begin{tabular}{|c|c|c|c|}
\hline & \multicolumn{3}{|c|}{ Microcell } \\
\hline & $\sigma_{\tau}$ & $\sigma_{\theta}$ & $\sigma_{\varsigma}$ \\
\hline $\begin{array}{c}\text { Proposed Algorithm } \\
(N=100)\end{array}$ & $5 \mathrm{ps}$ & $5^{\circ}$ & $200 \mu \mathrm{Hz}$ \\
\hline $\begin{array}{c}\text { Proposed Algorithm } \\
(N=200)\end{array}$ & $20 \mathrm{ps}$ & $7^{\circ}$ & $600 \mu \mathrm{Hz}$ \\
\hline
\end{tabular}

The quantities in Tables II and III are found empirically by assuming that only one of the measurements at each time step has errors, while the other two are perfect. For each column, only the type of measurement stated in row 2 (i.e., TOA, AOA, or DS) has i.i.d. zero mean Gaussian noise with a standard deviation given by the table entry and the rest of measurements are perfect. For example, in the third row and second column of Table II, the value of $1.3 \mathrm{ps}$ for $\sigma_{\tau}$ means that the TOA measurement must have a standard deviation below $1.3 \mathrm{ps}$ in order to achieve the desired positioning accuracy, assuming that the DS and AOA measurement errors are zero.

Table II shows the tolerable measurement errors for $N=$ 100 and Table III shows the tolerable measurement errors for $N$ $=200$. Note that the number of measurements $N$ is limited by whether the assumptions from Section II are valid (e.g., constant velocity, interacting with the same scatterers, etc.). As can be seen, the proposed algorithm meets the positioning requirement with TOA errors that are more than double the standard deviation of TOA errors for the algorithm from [2], AOA errors that are almost double the standard deviation of AOA errors for the algorithm from [2], and DS errors that are an order of magnitude more than the standard deviation of DS errors for the algorithm from [2]. Furthermore, using more measurements makes both algorithms less sensitive to the measurement errors. Finally, both algorithms are very sensitive to TOA and DS errors, especially for low speeds. However, both algorithms are not sensitive to AOA errors due to the averaging of AOA measurements (see page 2).
There are two reasons why the proposed algorithm requires less accurate measurements than the algorithm from [2] to achieve a specific level of accuracy for the MS position estimate. The first reason is that in the second step of each algorithm (when the problem is that of a conventional TOA positioning problem), the algorithm from [2] uses LS whereas the proposed algorithm uses the iterative technique based on Taylor Series expansion around an initial MS position estimate (i.e., nonlinear LS). It has been shown that the LS method has lower accuracy than nonlinear LS methods [6]. The second reason is that in the first step of each algorithm (when the location of the scatterers is estimated), the proposed algorithm estimates the position of the scatterers more accurately than the algorithm from [2]. For example, in a microcellular environment with $\left[\begin{array}{ll}v_{x} & v_{y}\end{array}\right]^{\mathrm{T}}=[10 \mathrm{~m} / \mathrm{s} 18 \mathrm{~m} / \mathrm{s}]^{\mathrm{T}}, N=200$, and measurement errors on all three types of measurements simultaneously $\left(\sigma_{\tau}=10 \mathrm{ps}, \sigma_{\theta}=10^{\circ}\right.$, and $\left.\sigma_{\varsigma}=1 \mu \mathrm{Hz}\right)$, the proposed algorithm estimation error per scatterer has a mean of $26.6 \mathrm{~m}$ and standard deviation of $48.3 \mathrm{~m}$ whereas the algorithm from [2] has a mean of $65.6 \mathrm{~m}$ and standard deviation of $548.9 \mathrm{~m}$. This poor performance for the algorithm from [2] is due to approximately $8 \%$ of the scatterer position estimates are in error of more than $2 \mathrm{~km}$, whereas the largest error for the proposed algorithm is $570 \mathrm{~m}$. Upon removing the poor estimates (i.e., scatterer position estimates in error of more than $2 \mathrm{~km}$ ), the proposed algorithm still outperforms the algorithm from [2]: the proposed algorithm estimation error per scatterer is the same as before whereas the algorithm from [2] now has a mean of $35.0 \mathrm{~m}$ and standard deviation of $73.0 \mathrm{~m}$. 
It should be noted that since the values in Tables II and III were obtained assuming two of the three measurements are error free, they provide a somewhat overly optimistic view of the algorithm's performance (although they do show that the proposed method is better than that in [2]). For example, one really needs TOA accuracy better than $1.3 \mathrm{ps}$ (third row and second column of Table II) in order to achieve the desired accuracy when the AOA and DS measurements have errors.

In the second set of results (see Table IV), the algorithm is evaluated based on what measurement errors are tolerable that still satisfy the positioning requirement of less than $50 \mathrm{~m}$ accuracy in $67 \%$ of positioning attempts for $1 \mathrm{BS}$ and $S=2$ scatterers. In this case, the algorithm from [2] has poor estimation performance and is unable to satisfy the positioning requirement even with perfect measurements. The results from Tables II through IV show that more accurate measurements are required in the case of 2 scatterers when compared to the case of 3 scatterers: TOA errors that are less than one order of magnitude lower than the standard deviation of the 3 scatterer case, AOA errors that are less than one-fourth the standard deviation of the 3 scatterer case, and DS errors that are less than one-fifth the standard deviation of the 3 scatterer case.

Lastly, Fig. 2 gives an example of what measurement errors are tolerable when all three measurement errors are present simultaneously. In these results, the standard deviation of the AOA measurement error is constant at $\sigma_{\theta}=2^{\circ}$ (since it has already been seen that the algorithm is not sensitive to AOA errors) and the standard deviations of the TOA and DS measurement errors are varied. The shaded region represents the TOA and DS errors that still satisfy the positioning requirement of less than $50 \mathrm{~m}$ accuracy in $67 \%$ of positioning attempts for $1 \mathrm{BS}$ and $S=2$ scatterers in a microcellular environment with $N=200$. Notice that all of the tolerable measurement errors in Fig. 2 are less than their corresponding values in the last row of Table IV, which is expected because the presence of all three measurement errors simultaneously requires more accurate measurements to achieve the same positioning accuracy compared to when only one of the three measurement errors is present (as discussed two paragraphs earlier).

\section{CONCLUSION}

In this paper, a novel LS algorithm was presented to estimate the location of a MS experiencing purely NLOS communication with at least one BS by using the TOA, AOA, and DS measurements collected at the BS. Simulation results were provided to demonstrate the estimation accuracy in picocellular, microcellular, and macrocelluar environments. It was shown that the proposed algorithm requires less accurate measurements than existing comparable methods to achieve a specific level of accuracy for the MS position estimate. Furthermore, it was seen that a 2-d location estimate can be produced with as little as $1 \mathrm{BS}$ and 2 scatterers (or comparably, 2 BS, each with 1 scatterer).

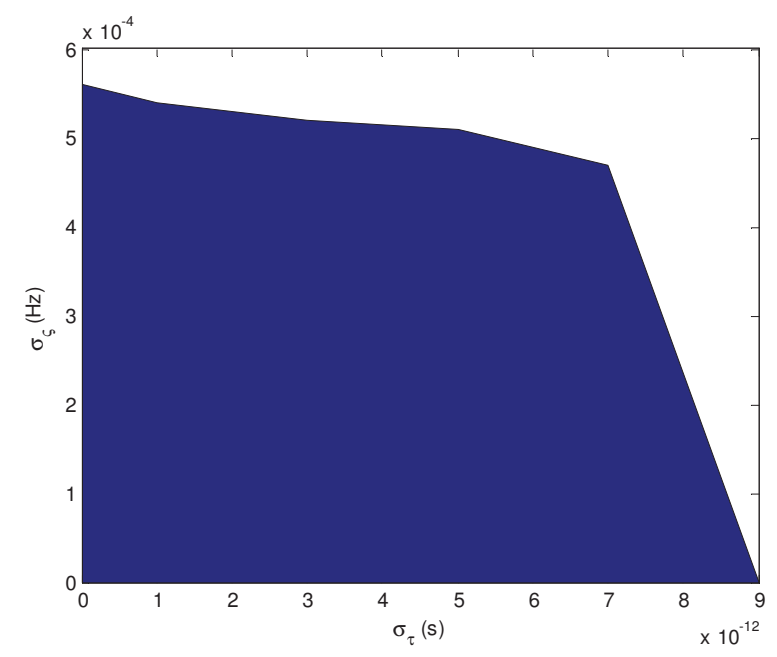

Fig. 2. Tolerable TOA and DS measurement errors (microcell, $N=200$, $\left.\sigma_{\theta}=2^{\circ}\right)$

Even though the proposed algorithm requires accurate TOA and DS measurements, it is the authors' hope that these results will help further research into a robust radio frequency localization method in cellular communication systems. As a comment on the feasibility of these accurate measurements, one can look at the fundamental bound on TOA estimation accuracy derived by Weiss and Weinstein, which states that the estimation accuracy can improve as the carrier frequency of the signal increases if the signal-to-noise ratio is large enough, in addition to the time-bandwidth product [7]-[9].

\section{REFERENCES}

[1] N. Khan, M. Simsim, P. Rapajic, "A Generalized Model for the Spatial Characteristics of the Cellular Mobile Channel," IEEE Trans. Veh. Technol., vol.57, no.1, pp.22-37, Jan. 2008.

[2] K. Papakonstantinou, D. Slock, "Hybrid TOA/AOD/Doppler-Shift localization algorithm for NLOS environments," Proc. IEEE PIMRC, Tokyo, Japan, Sept. 2009, pp.1948-1952.

[3] J. Chen, F. Jiang, A. Swindlehurst, J. Lopez-Salcedo, "Localization of mobile equipment in radio environments with no line-of-sight path," Proc. IEEE ICASSP, Vancouver, BC, Canada, May 2013, pp.4081-4085.

[4] E. Kaplan, C. Hegarty, "Fundamentals of Satellite Navigation" in Understanding GPS: Principles and Applications, 2nd ed. Norwood: Artech House, 2006, ch.2, sec.4.2, pp.54-58.

[5] The FCC, "Wireless 911 location accuracy requirements, second report and order," FCC 10-176, 2010.

[6] R. Zekavat, R. Buehrer, "Source Localization: Algorithms and Analysis" in Handbook of Position Location: Theory, Practice and Advances, 1st ed. Hoboken: John Wiley \& Sons, 2012, ch.2, sec.3, pp.34-55.

[7] A. Weiss, E. Weinstein, "Fundamental limitations in passive time delay estimation-part i: Narrow-band systems," IEEE Trans. Acoust., Speech, Signal Process., vol.31, no.2, pp.472-486, Apr. 1983.

[8] E. Weinstein, A.Weiss, "Fundamental limitations in passive time-delay estimation-part ii: Wide-band systems," IEEE Trans. Acoust., Speech, Signal Process., vol.32, no.5, pp.1064-1078, Oct. 1984.

[9] P. Bidigare, U. Madhow, R. Mudumbai, D. Scherber, "Attaining fundamental bounds on timing synchronization," Proc. IEEE ICASSP, Kyoto, Japan, Mar. 2012, pp.5229-5232. 